\title{
Indocyanine green angiographic findings in serpiginous choroidopathy
}

Clinica Oculistica dell' Università di Ancona, Nuovo Ospedale

Regionale di Torrette, Ancona, Italy

A Giovannini

C Mariotti

E Ripa

B Scassellati-Sforzolini

Correspondence to: A Giovannini, Clinica

Oculistica dell' Università di Ancona, Nuovo Ospedale Regionale di Torrette, 60020 Ancona, Italy.

Accepted for publication 11 March 1996

\author{
A Giovannini, C Mariotti, E Ripa, B Scassellati-Sforzolini
}

\begin{abstract}
Aims-Analysis of the choroidal findings in patients affected by serpiginous choroidopathy (SC).

Methods-Thirteen patients (23 eyes; 11 males and two females; age range 50-68 years; mean age 59.1 years) affected by $\mathrm{SC}$ were examined with fluorescein angiography (FA) and indocyanine green angiography (ICGA). The follow up period was 7-33 months.

Results-Using ICGA the disease could be divided into the the following stages: (1) subclinical or choroidal stage (hypofluorescent lesions without FA evidence); (2) active stage (with ICGA and FA evidence); (3) subhealing stage (slight late hyperfluorescent lesions with ICGA, with no evidence on FA); (4) inactive or healed stage (hypofluorescent areas with ICGA and hyperfluorescent areas with FA).

Conclusions-Although FA showed a clear distinction between active and healed stages, ICGA allowed a greater subdivision of the disease. In particular, ICGA allowed: (1) better staging of SC, revealing choroidal alterations when there was no ophthalmoscopic or FA evidence; (2) better identification of the active lesions which appear to be larger at the choroidal level in comparison with the corresponding retinal lesions; and (3) revealed a persistence of choroidal activity even when the signs of retinal activity had disappeared. Thus, ICGA should be a particularly useful clinical and therapeutic monitoring tool of SC.
\end{abstract}

(Br F Ophthalmol 1996;80:536-540)

Serpiginous choroidopathy (SC) usually extends from the optic disc outwards in all direction $\mathrm{s}^{1-3}$; initial spreading from the macular area is occasional and at first free of any peripapillar activity (serpiginous maculopathy). ${ }^{45} \mathrm{SC}$, known also as geographic helicoid peripapillary choroidopathy ${ }^{2}$ is a chronic, recurrent, progressive, usually bilateral disorder, that extends from the choroid to the retinal pigment epithelium (RPE) and ultimately to the retina. In the acute stage it is common to observe the concomitant expression of new and old lesions. The active lesions are greyish white or yellow in colour, with faint edges and are sometimes associated with a serous detachment of the neuroepithelium and/or less frequently of the RPE. ${ }^{6} \mathrm{SC}$ can be further complicated by choroidal neovascularisation (CNV), usually occurring at the edge of an old lesion, which may represent a further cause of visual impairment in such patients. Other findings include retinal vasculitis, ${ }^{7}$ generally at the site of an active lesion and venous branch occlusion. ${ }^{8}$ The healed stage is characterised by well defined, irregularly pigmented atrophic chorioretinal areas. Visual prognosis of SC depends on the extent of foveal involvement.

The active lesions are hypofluorescent during the early phases of fluorescein angiography and hyperfluorescent in the late phases with late diffusion. The healed lesions, on the other hand, initially appear as an area of hypofluorescence, along with clearly visible residual large choroidal vessels, secondary to choriocapillaris and RPE atrophy. As a result of fluorescein spreading from the choriocapillaris and scleral staining, the area of healing becomes hyperfluorescent, with well defined edges.

The causes of this disorder are still under investigation. However, there are theories suggesting that either inflammatory ${ }^{-11}$ or vascular factors are involved. ${ }^{11}{ }^{12}$ Data collected from the limited histopathological studies performed to date ${ }^{1013}$ tend to favour the inflammatory hypothesis; indeed extensive lymphocyte choroidal infiltrates were found at the margin of thelesion, but a coagulation disorder (increase in factor VIII) has been observed in some cases. ${ }^{13}$ The distribution of the lesions may suggest a choroidal arterial occlusion and its vascular character would make it possible to unify the two theories.

Indocyanine green (ICG) angiography allows a better investigation of the choroidal circulation: ICG has the highest point of absorption and the maximum emission in the near infrared, and so allows us to overcome the screen represented by the RPE and xanthophyll. Good visualisation of the vascular choroidal system (even if there are still limits in the exploration of the choriocapillaris) is due to the fact that $98 \%$ of ICG is linked to plasma proteins, with a consequent poor diffusion outside the choroidal and/or retinal normal vessel walls. ICG angiography (ICGA), because of the possibilities it offers and the choroidal nature of SC, is undoubtedly helpful in assessing the possible pathogenesis of the disease.

The aim of our study was to compare fluorescein angiography (FA) and ICGA choroidal findings in order to improve the staging and the evaluation of the therapeutic response of SC.

Materials and methods

With high definition videoangiography (Topcon IMAGEnet H1024) we examined 13 
patients affected by SC ( 23 eyes; 11 males and two females; age 50-68 years; mean age 59.1 years): six patients (nine eyes) at the active stage viewed with ophthalmoscopy (yellow plaque at the level of the RPE) and FA, seven patients (14 eyes) at the inactive stage. The patients were followed up for 7-33 months.

All patients underwent complete ophthalmic examination: colour and red-free fundus photography, FA, and ICGA. ICGA was performed using $25 \mathrm{mg} /$ patient of ICG (Cardiogreen, Hynson, Westcott and Dunning, Inc, Baltimore, MD, USA) injected into a peripheral arm vein which was followed immediately by a $5 \mathrm{ml}$ flush of sterile saline via a three way stopcock.
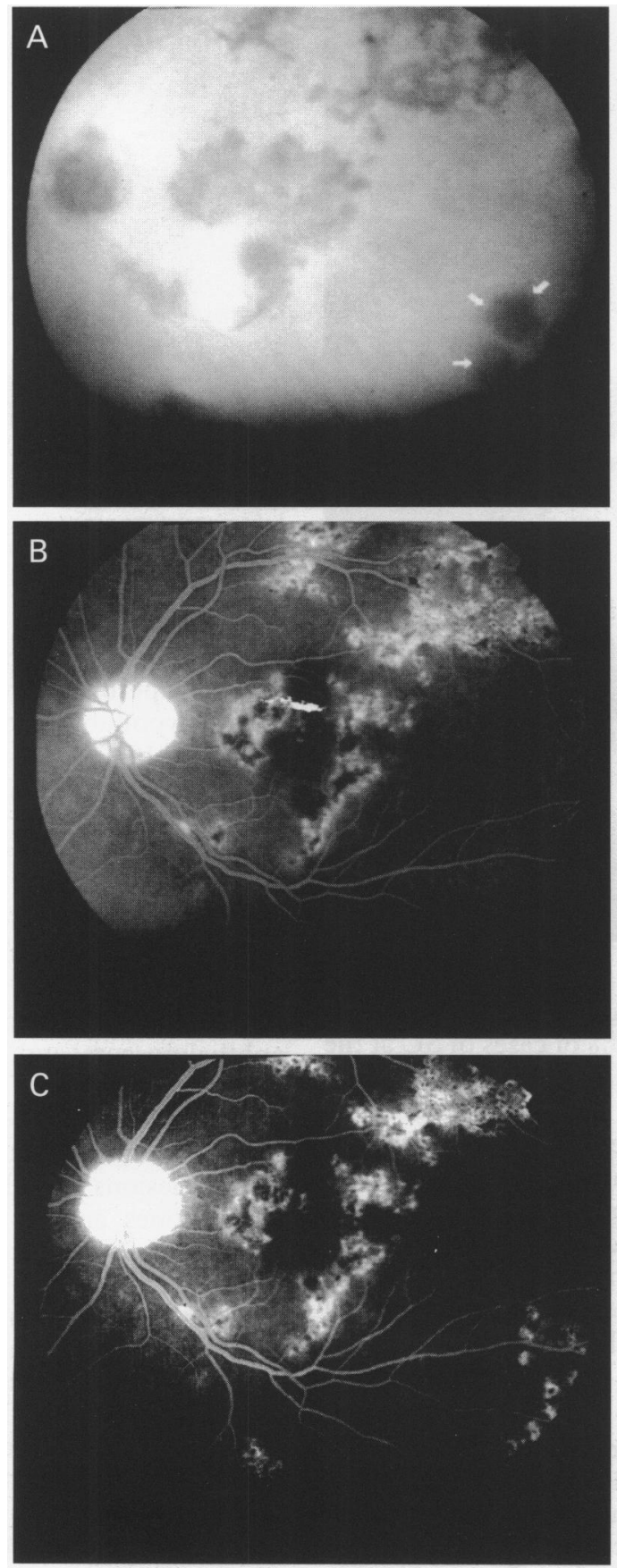

Figure 1 Subclinical stage. (A) Indocyanine green angiography (ICGA) shows hypofluorescent choroidal lesions with faint edges (arrows). (B) The lesions are not evident on fluorescein angiography (FA). (C) Two month later the lesions are seen even on FA, in an area corresponding to the choroidal lesions seen with ICGA.

\section{Results}

Of the 23 eyes examined, nine presented signs of ophthalmoscopic and FA activity. In four of them we observed more than one recurrence (one case presented two recurrences, two cases three recurrences, and one case four recurrences).

FA signs of activity (early hypofluorescence with late leakage of the focus) have been well described by Schatz et al, ${ }^{13}$ Hamilton and Bird, ${ }^{14}$ and Laatikainen and Erkkila. ${ }^{15}$

The findings provided by ICGA in the active stage, after the observation of all recurrences, can be summarised as follows:

(1) In seven eyes we found a correspondence between ICG and FA findings, characterised by early hypofluorescent areas with late hyperfluorescence and faint edges.

(2) In 10 eyes ICGA showed in all angiographic phases an area of hypofluorescence with ill defined margins and a size greater than that seen in FA.

(3) A third pattern of activity was observed only with ICGA: a hypofluorescent area (distant from the area observed in FA) with ill defined margins in all phases (three eyes).

(4) ICGA showed, in our series, choroidal hyperfluorescence only in the late phases, after the active focus appeared to have healed when viewed with an ophthalmoscope and FA (two eyes). In these cases only ICGA revealed choroidal permeability alterations.

ICGA alsorevealed delayed choroidal filling (a time longer or equal to 8 seconds from the
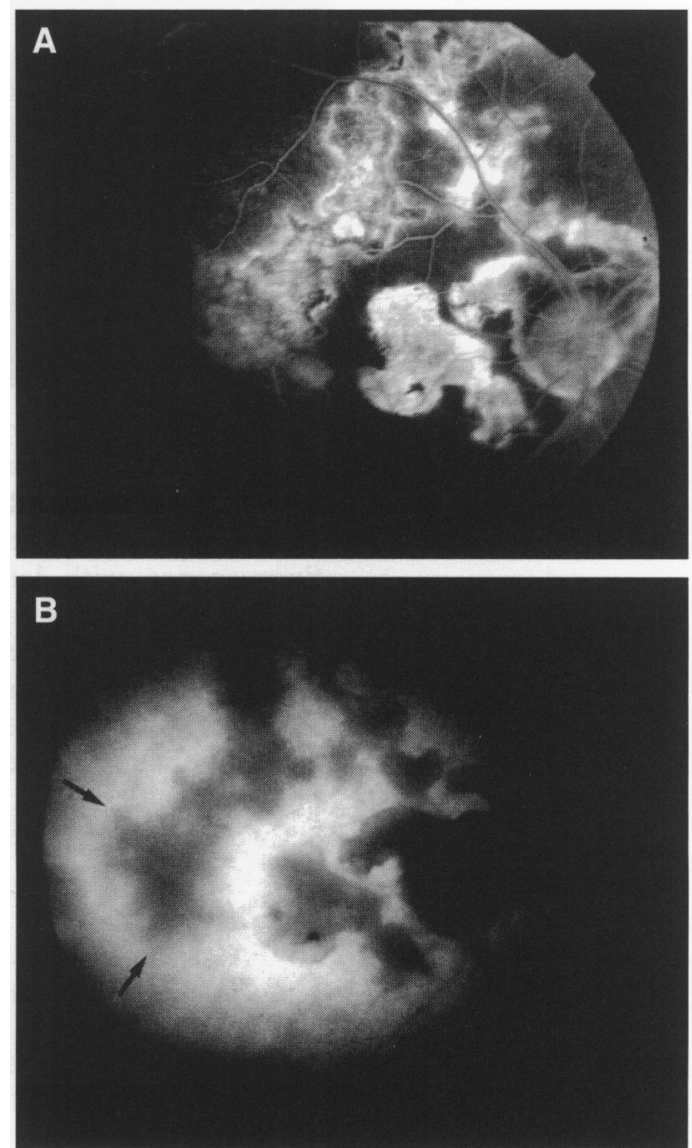

Figure 2 Active stage. (A) Fluorescein angiography shows an active focus, hyperfluorescent in the late phases (arrow). (B) In the indocyanine green angiograms the active area appears hypofluorescent with faint edges and no late hyperfluorescence (arrows). 

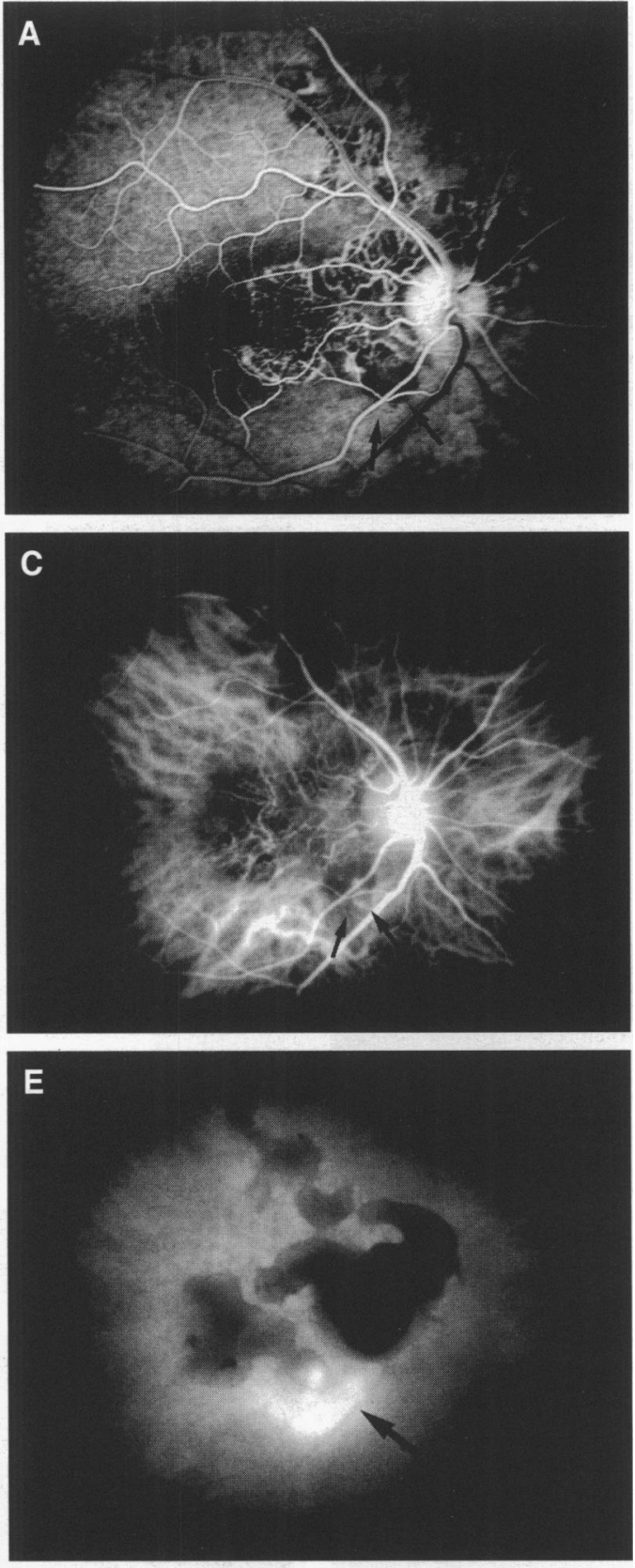

moment of the choroidal blush till the full choroidal filling ${ }^{16}{ }^{17}$ in $42.1 \%$ of cases of $S C$ at the active stage. Moreover, ICGA showed hyperfluorescence with late leakage of the active lesions in $47.4 \%$ of cases secondary to choroidal permeability alterations. We observed a reduction or regression of activity of the initially active lesions after systemic steroids $(1 \mathrm{~g}$ intravenous methylprednisolone for 5 consecutive days) in 11 eyes presenting signs of activity or recurrences threatening the fovea.

\section{Discussion}

If FA showed an unequivocal distinction between the active and the inactive stages of SC, ICGA seemed to enable us to make a more accurate analysis of the disease. In ICGA the active focus appeared as a hypofluorescent area with faint edges, visible from the early phases to the intermediate and late phases of the angiography. This image was frequently accompanied by a hyperfluorescent halo varying in intensity, with diffusion in the late angio-
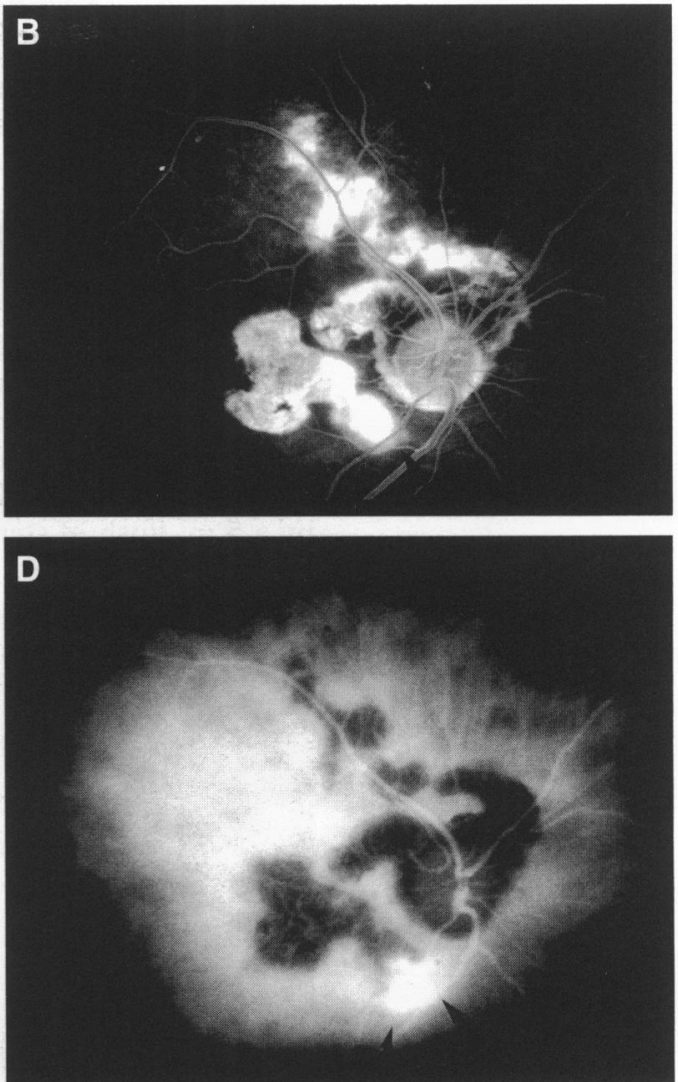

Figure 3 Active stage. (A) (B) Fluorescein angiography reveals the classic early hypofluorescence with late hyperfluorescence of the active focus (arrows). (C) (D) The behaviour of the active focus in the indocyanine green angiogram is comparable (arrows). (E) Note the marked late leakage (arrow).

graphic phases, a possible sign of alteration in choroidal permeability. The area of healing was always hypofluorescent with well defined margins in all ICGA phases.r

The comparison of the data obtained from FA and ICGA of our patients allow us to classify SC into four distinct stages.

(1) The subclinical stage (choroidal stage of the disease) is characterised by hypofluorescent lesions with faint edges, the manifestation of choroidal changes was detectable only with ICGA; the RPE remains unaffected, indeed ophthalmoscopy and FA indicate that no such lesions are present. This aspect may continue for a prolonged period of time without additional manifestations. During the follow up, new lesions may also be visible distant from former ones and on FA, alterations can be detected at the same site previously seen on ICGA (Fig 1).

(2) At the active stage, when the lesion damages the RPE and therefore becomes evident on ophthalmoscopic and FA examination (retinal stage), two ICG angiographic patterns are evident: (a) at the active stage, and in the presence of the aforementioned sign of activity (early hypofluorescence with late leakage), the ICGA reveals active areas of hypofluorescence which are similar in size, but are nevertheless usually larger compared with those observed in FA. No late hyperfluorescence is evident (Fig 2); (b) occasionally the classic FA early hypofluorescence with late leakage of the active focus, corresponds to an early ICGA hypoflu- 

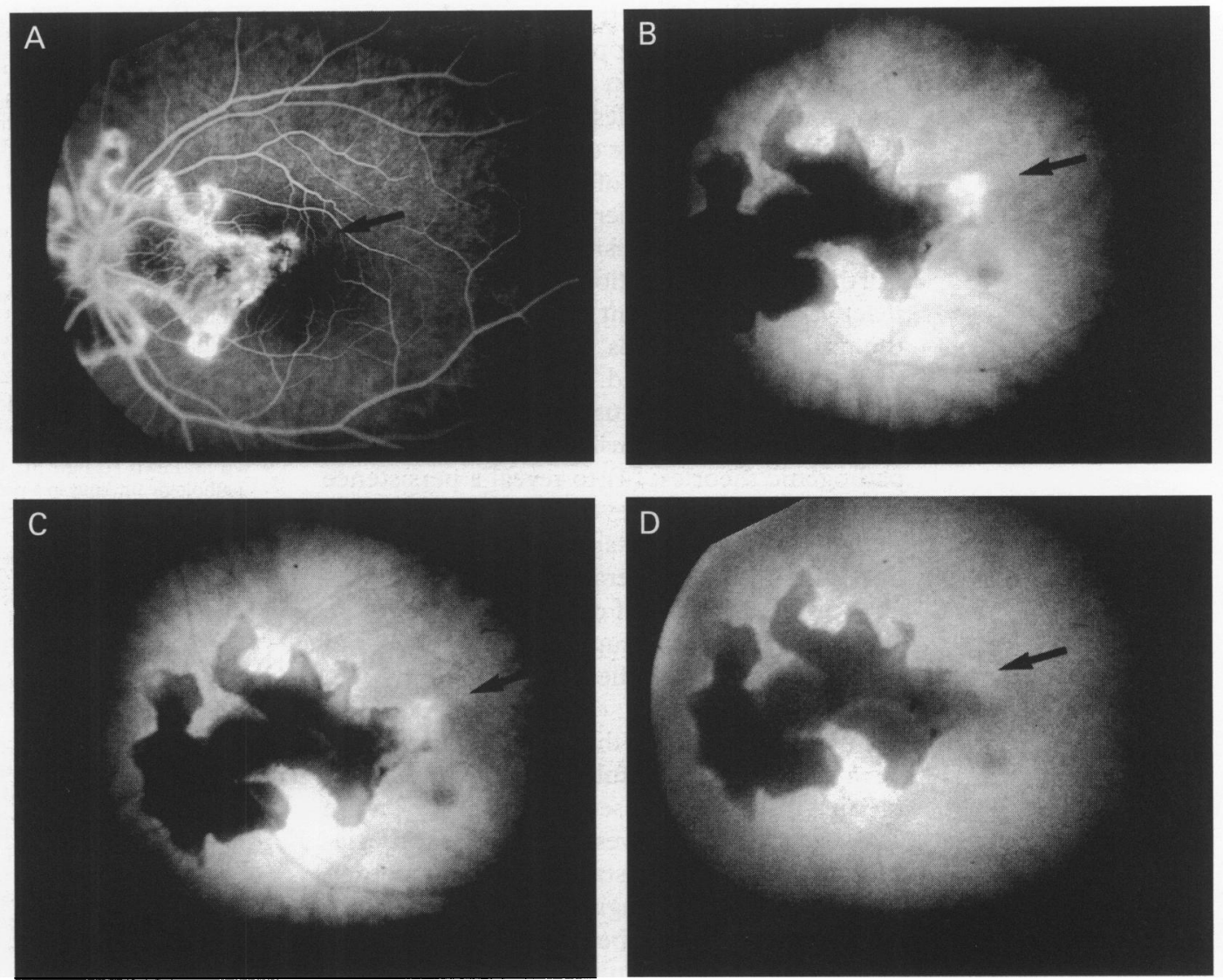

Figure 4 Third stage. (A) Fluorescein angiography reveals no activity (arrow). (B) (C) Indocyanine green angiography shows a hyperfluorescent halo with slight late leakage (arrows). (D) After 2 months of systemic steroids a regression of the hyperfluorescence can be observed (arrow).

oescence with faint edges and is associated with a marked hyperfluorescence with evident diffusion in the la $a^{*}$ - mases. These manifestations of altered permeability of the choroidal vessels are sign of increased aggressiveness of the disease (Fig 3).

(3) ICGA makes it possible to identify a subhealing stage characterised by activity which persists at the level of the choroid, after the focus itself appears to have healed at the level of the RPE. In such cases choroidal permeability alterations, which are usually adjacent to a recently healed focus, can be detected only by ICGA. These anomalies are characterised by a slight late diffusion without FA evidence. Systemic steroid therapy can cause the regression of such signs of choroidal activity (Fig 4)

(4) The inactive or healed stage presents as an area of hypofluorescence with clearly defined margins; this hypofluorescence becomes more marked in the late phases. Within the area itself, images of greater hypofluorescence emerge indicating irregular pigmentation of the atrophic zones, together with the remaining patent choroidal vessels (Fig 5). Note that the extension of the healed area is usually smaller than that at the active stage. After steroid therapy a reduction in the extent of the inactive hypofluorescent area compared with the initial active lesions is visible.

In the light of our observations and evidence provided by FA and ICGA we present a new classification of SC. ICGA made it possible to observe in more detail the various aspects of the disorder than did FA. In particular it
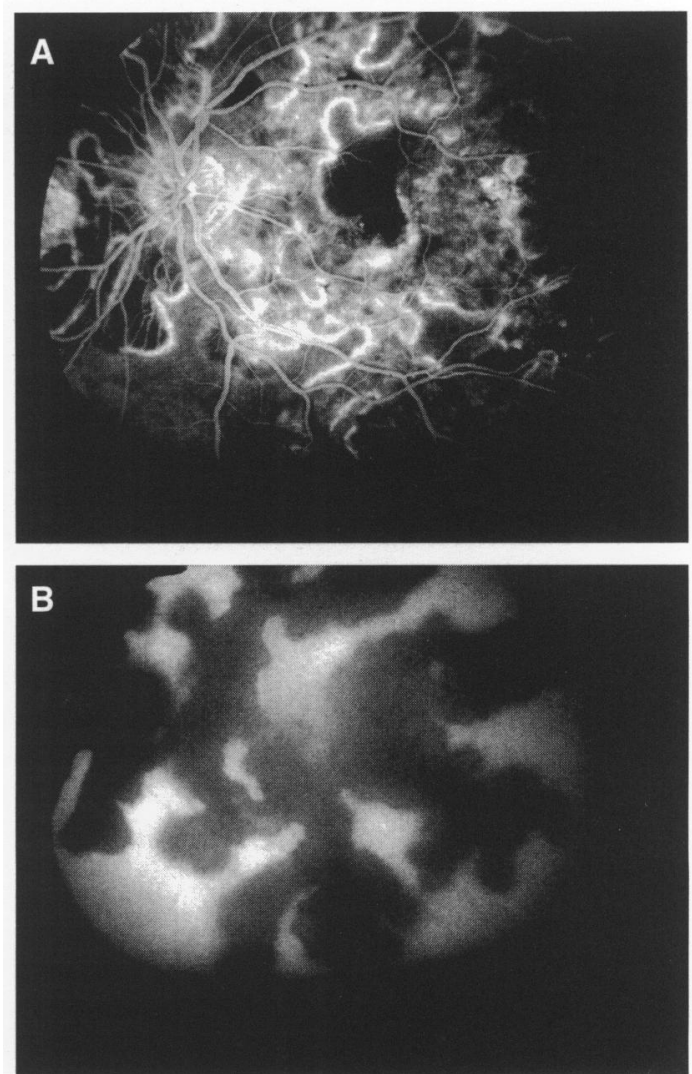

Figure 5 Healed stage. (A) Fluorescein angiographic picture. (B) Indocyanine green angiography displays a hypofluorescent lesion with well defined margins in all angiographic phases.

allowed: (1) better staging of SC, revealing choroidal alterations without ophthalmoscopic 
or FA evidence; (2) better identification of the active lesions, which appear to be larger at the level of the choroid in comparison with the corresponding retinal lesions; (3) to point out, at the active stage of the disease, a delayed choroidal filling in $42.1 \%$ of cases; and to indicate signs of vasculitis (hyperfluorescence with late leakage) of the active lesions in $47.4 \%$ of cases. Furthermore, a reduction in the extent of the inactive hypofluorescent areas, compared with the initial active lesions, after systemic steroid therapy was observed. All these elements would therefore allow us to hypothesise a correlation between occlusive and inflammatory pathogenic theories; (4) to reveal a persistence of choroidal activity even when the signs of retinal activity have disappeared. The observation of choroidal alterations without retinal changes could be proof of the choroidal nature of the affection, as is supposed on the basis of histopathological studies by $\mathrm{Gass}^{13}$ and Wu et al. ${ }^{9}$

In consideration of the above, ICGA could be a particularly useful tool for the clinical monitoring of SC and seems to provide a more sensitive method of investigation and appreciation of the various stages of the disorder. Moreover, the ICG angiographic signs of subclinical activity could be helpful in developing more accurate therapeutic schemes.
1 Weiss H, Annesley WH Jr, Shields JA, Tomer T, Christopherson $\mathrm{K}$. The clinical course of serpiginous choroidopathy. Am f Ophthalmol 1979;87:133-42.

2 Schatz H, Maumenee AK, Patz A. Geographical helicoid peripapillary choroidopathy: clinical presentation and fluoperipapillary choroidopathy: clinical presentation and fluoOtolaryngol 1974;78:747-61.

3 Chisholm IH, Gass JDM, Hutton WL. The late stage of serpiginous (geographic) choroiditis. Am f Ophthalmol 1976; $82: 343-51$.

4 Hardy RA, Schatz H. Macular geographic helicoid choroidopathy. Arch Ophthalmol 1987;105:1237-42.

5 Mansour JM, Jampol LM, Packo KH, Hrisomalos NF. Macular serpiginous choroiditis. Retina 1988;8:125-31.

6 Wojno T, Meredith TA. Unusual findings in serpiginous choroiditis. Am f Ophthalmol 1982;94:650-5.

7 Blumenkranz MJ, Gass JDM, Clarkson JG. Atypical serpiginous choroiditis. Arch Ophthalmol 1982;100:1773-5.

8 Erkkila $\mathrm{H}$. Laatikainen $\mathrm{L}$, Jokinen $\mathrm{E}$. Immunological studies of serpiginous choroiditis. Graefes Arch Clin Exp Ophthalmol of serpiginous ch

9 Wu JS, Lewis H, Fine SL, Grover DA, Green WR. Clinicopathologic findings in a patient with serpiginous choroiditis and treated choroidal neovascularization. Retina 1989;9: 292-301.

10 Becquet F, Cohen SY, Coscas G. Choroidite serpigineuse. $\mathcal{Y}$ Fr Ophtalmol 1992;4:288-298.

11 King DG, Grizzard WS, Sever RJ, Espinoza L. Serpiginous choroidopathy associated with elevated factor VIII. Von Willebrand factor antigen. Retina 1990;10:97-101.

12 Gass JDM. Specific choroidal diseases causing disciform macular detachment. In: Gass JDM, ed. Stereoscopic atlas of macular detachment. In: Gass JDM, ed. Stereoscopic atlas of macular diseases: diagnosis

13 Schatz H, Maumenee AK, Patz A. Geographic helicoid peripapillary choroidopathy. Trans Am Acad Ophthalmol Otolaryngol 1974;78:747-61.

14 Hamilton AM, Bird AC. Geographical choroidopathy. $\mathrm{Br} \mathcal{F}$ Ophthalmol 1974;58:784-97.

15 Laatikainen L, Erkkila $\mathrm{H}$. Serpiginous choroiditis. $\mathrm{Br} f$ Ophthalmol 1974;58:777-83.

16 De Laey JJ. Fluoro-angiographic study of the choroid in man. Bull Soc Belge Ophtalmol 1977;174:1-217.

17 Giovannini A, Mariotti C, Ripa E, Scassellati Sforzolini B Tiovannini A, Mariotti C, Ripa E, Scassellati Sforzolini B,
Tittari Choroidal filling in age-related macular degeneration: indocyanine green angiographic findings. Ophthalmologica 1994;208:185-91. 